\title{
Gaining Weight: How Media Exposure, Socioeconomic Status, Sedentary Lifestyle and Dietary Habits Work Together - A Secondary Analysis of a Nationally Representative Study
}

\author{
Taejin Jung* \\ 7 Lanigan Hall, SUNY Oswego, Oswego, NY 13126, USA
}

\begin{abstract}
Obesity is a complex phenomenon without a single cause that needs comprehensive understanding and integrated intervention. However, few researches have considered the media exposure, SES, lifestyle factors and their contributions to the national problem of overweight and obesity comprehensively. To study the determinants of adults being overweight or obese, a secondary analysis with national representative sample (ABC News/Time Magazine Obesity Poll) was performed. Respondents were asked to rate their media exposures, behaviors of healthy diets and physical activities in their lives. The research results reaffirmed that sedentary lifestyle (e.g., hours of watching television and physical inactivity) in combination with dietary behavior of eating fat condensed food (e.g., frequent visits of fast food restaurant) have increased probabilities to be overweight and obese.
\end{abstract}

\section{INTRODUCTION}

The number of overweight people and obesity levels has increased in every state, every year, for decades (Pastor, Makuc, Reuben, \& Xia, 2002). The prevalence of both overweight people and obesity has continuously increased across gender, all ages, all racial/ethnic groups, and all education levels (Mokdad, 2003). From 1960 to 2000, the prevalence of overweight people $(25 \leq \mathrm{BMI}<30)$ has risen from 31.5 to 33.6 percent in U.S. adults aged 20 to 74 (Pastor, Makuc, Reuben, \& Xia, 2002) and obesity (BMI $\geq 30$ ) during the same period of time has doubled from 13.3 to 30.9 percent, with most of this rise occurring in the past 20 years (Flegal, Carroll, Ogden, \& Johnson, 2002). Unhealthy dietary behavior and sedentary lifestyle are accountable for at least 300,000 deaths each year (McGinnis \& Foege, 1993). It is estimated that being overweight and overweight-related diseases cost the health care industry 100 billion dollars annually (Harver-Berino, Pintauro, \& Gold, 2002).

The latest study based on the nationally representative sample of U.S. adults estimates that about 112,000 deaths are associated with obesity each year in the United States (Flegal, Graubard, Williams, \& Gail, 2005). According to an American Cancer Society study, when controlling for smoking, there was a graded increase in mortality with an increasing body mass index (BMI) that is especially significant at a BMI of 27 to 29.9 (Manson, Stamfer, Hennekens, \& Willett, 1987). A health profession study also indicated that there is a graded increase in mortality from heart disease associated with increasing degrees of weight gain (Willett et al., 1995). Likewise, research dealing with weight loss found that it affects a number of risk factors. Losing 5-10 percent of a person's body weight leads to changes in blood pressure and triglyceride levels and usually a 10 percent weight loss is

Address correspondence to this author at the 7 Lanigan Hall, SUNY Oswego, Oswego, NY 13126, USA; Tel: 315.312.3527; Fax: 315.312.5658; E-mail: jung@oswego.edu sufficient to see significant improvement in risk factors (Sjostrom, Lissner, \& Sjostrom, 1997).

The American Obesity Association cites several factors as contributors to obesity including an increase in television viewing, computer use and playing video games along with other factors such as a sedentary occupational lifestyle (AOA, 2004). An observational cohort study of approximately one million persons following 14 years reported a linear relationship between BMI and mortality (Calle, Thun, Petrelli, Rodriguez, \& Heath, 1999). The interdependence of dietary behaviors and inactivity patterns as risk factors for a variety of diseases which can be a result of obesity is associated with an increased risk of cardiovascular disease, certain cancers, diabetes, depression, discrimination, weight-related bias and other various physical, psychological and social morbidities (Chambliss, Finley, \& Blair, 2004; Pi-Sunyer \& Xavier, 1999; Van Cauter \& Spiegel, 1999).

Gaining weight is a complex phenomenon without a single cause that needs a comprehensive understanding and integrated intervention. However, there have been few researches confirming the main factors and the degree to which they contribute to the national problem of gaining weight with a nationally representative study. Through the national study of $\mathrm{ABC} / \mathrm{Time}$ magazine poll, this research tries to deal with the main causing factors and their respective contributions of gaining weight on four aspects; media exposure, socioeconomic factors, sedentary life style (physical inactivity) and dietary behaviors.

So, the principal research question to be answered is how individual media exposure, socioeconomic status, sedentary life style and eating behavior have effects on gaining weight independently and in combination with each other. Respondents were asked to rate their amount of media exposure (e.g., TV and Internet), the behavior of a healthy diet and physical exercise in their lives. The results of this research showed that sedentary lifestyles (e.g., less physical activities and hours of watching television) and dietary behavior of 
eating fatty condensed foods (e.g., frequent visits to fast food restaurants) increase the probability of being overweight and/or obese.

\section{The Influence of SES on Gaining Weight}

It is well documented that people of lower socioeconomic levels are significantly more likely to consume highly condensed fatty foods, to lead a sedentary lifestyle, to smoke cigarettes, and to be overweight (Winkleby, Fortmann, \& Barrett, 1990). The socioeconomic status gradient in adult health has been documented throughout industrialized nations (Adler, Boyee, \& Chesney, 1994). Lower household income and lower parental education respectively were associated with obesity in a national sample of adolescents (Goodman, Slap, \& Huang, 2003). SES has been an underlying force that continues to create health disparities even in the changing presence of proximal risk factors. Link \& Phelan (1995) argue that SES and social support are likely fundamental causes of diseases because they embody access to important resources and affect multiple disease outcomes through multiple mechanisms. Access hypothesis also suggests that low socioeconomic status reduces educational opportunities, resulting in lower levels of knowledge and behavioral skills for weight loss. Among other things, economic constraints limit behavioral options (e.g., access to physical exercise facilities or quality food) (Levy, Fein, \& Stephenson, 1993).

\section{The Influence of Sedentary Life Style and Unhealthy Diet on Gaining Weight}

It has long been documented that daily health behavior or lifestyle factors - lack of regular exercise, sedentary lifestyle, inappropriate food consumption (based on caloric intake), and smoking - have been cited as the main indicators of being overweight or obese (McGinnis \& Foege, 1993). A report by a World Health Organization (WHO) Consultation on Obesity concluded that the fundamental causes of the obesity epidemic are sedentary lifestyles and high-fat, energy-dense diets (1998). It's true, especially in the United States, where less than one-third (31.8 percent) of the adult population gets regular leisure-time physical activity and about 10 percent of adults do not exercise at all in their leisure time (Barns \& Schoenborn, 2003). The majority of them are urbanites, city-dwellers who are more likely to consume an energy-dense diet that is high in saturated fat and refined carbohydrates. While the benefit of planned, long period exercise is well documented, there are real problems in generating a sustained commitment to regular exercise under the environment typical of a lack of time, pressure from work and other factors. In summary, the widespread declines in physical activity in combination with high fat intake are associated with rising rates of gaining weight.

Recent studies show that abuse of fast food as well as alcohol and cigarette smoking increases morbidity, mortality and healthcare costs (Strum \& Wells, 2001). French, Harnack, \& Jeffrey (2000) found that frequent patronage of fast food restaurants has shown positive associations with intake of total calories and percentage of fat and negative association of fiber intake. Unhealthy fast food consumption, together with an inactive lifestyle, has led some states' public health departments to consider fast food companies as partly responsible. Furthermore, it is also suggested that frequent exposure to fast food television advertisements may influence audiences' food choices to favor condensed fat or higher energy food (French, Story, \& Jeffrey, 2001).

A Henry J. Kaiser Family Foundation report showed that television watching is associated with adult obesity while television advertisements did have an impact on childhood obesity levels (Yach, 2004). Several studies indicate that there is a correlation between the amount of television viewing and the prevalence of obesity. Dietz and Gortmaker (1985) found that obesity is related to hours of television watching, with the prevalence of obesity increasing by approximately 2 percent for each additional hour of watching television.

Under the life course epidemiological perspective, insults can be a factor leading to excessive weight gain which occurs over the life course from conception, fetal, infant, childhood, adolescent and adult life. However, in adulthood, determining the risk factors for obesity associated with morbidities is even more important than identifying vulnerable or sensitive periods for weight gain. This research focuses directly on the behavioral and lifestyle factors related to weight gain in combination with socioeconomic status in the adult period, which includes decreased physical activity and unbalanced dietary intake. Even though there have been studies that blame SES (Goodman, Slap, \& Huang, 2003), high fat diets (Racette, Deusinger, Strube, Highstein, \& Deusinger, 2005; Saba, Vassallo, \& Turrini, 2000), physical inactivity (Caspersen, Pereira, \& Curran, 2000; MMWR, 2004), or media exposure (Dietz \& Gortmaker, 1985; Yach, 2004) on gaining weight respectively, few studies have investigated the combined contributions of main factors on gaining weight of US adults all at once. Moreover, this study contributes to the literature because it uses the national representative sample to reaffirm the relationships between main contributing factors of gaining weight and corresponding measure of body mass index. This study also reveals the probabilities to be overweight or obese through the suggested unhealthy life style with the most current nationally representative data set.

Overall, this research attempts to bring greater clarity to weight gain issue by addressing the following questions with the national representative data; (1) what is the relationship between SES and its effect on gaining weight? (2) what are the relative magnitudes of the effects of media exposure, SES, physical activity and eating habits on gaining weight in a hierarchical point of view?

\section{METHODS}

\section{Sample \& Study Design}

This study is a secondary analysis of data originally collected through the ABC News/Time Magazine Obesity Poll conducted on May 2004. A stratified, multistage area sample of adults age 18 and over living in the United States was selected for the study. A sample of households in the continental U.S. was selected via random digit dialing (RDD) procedure to insure that all possible listed and unlisted phone numbers were included with equal probability of selection. To compensate for the fact that women tend to be easier to reach, in house selection is stratified by gender, with interviewers asking to speak with the male household member 75 percent of the time and the female 25 percent of the time. 
Table 1. Demographic Characteristics of Study Variables $(\mathrm{N}=1,202)$

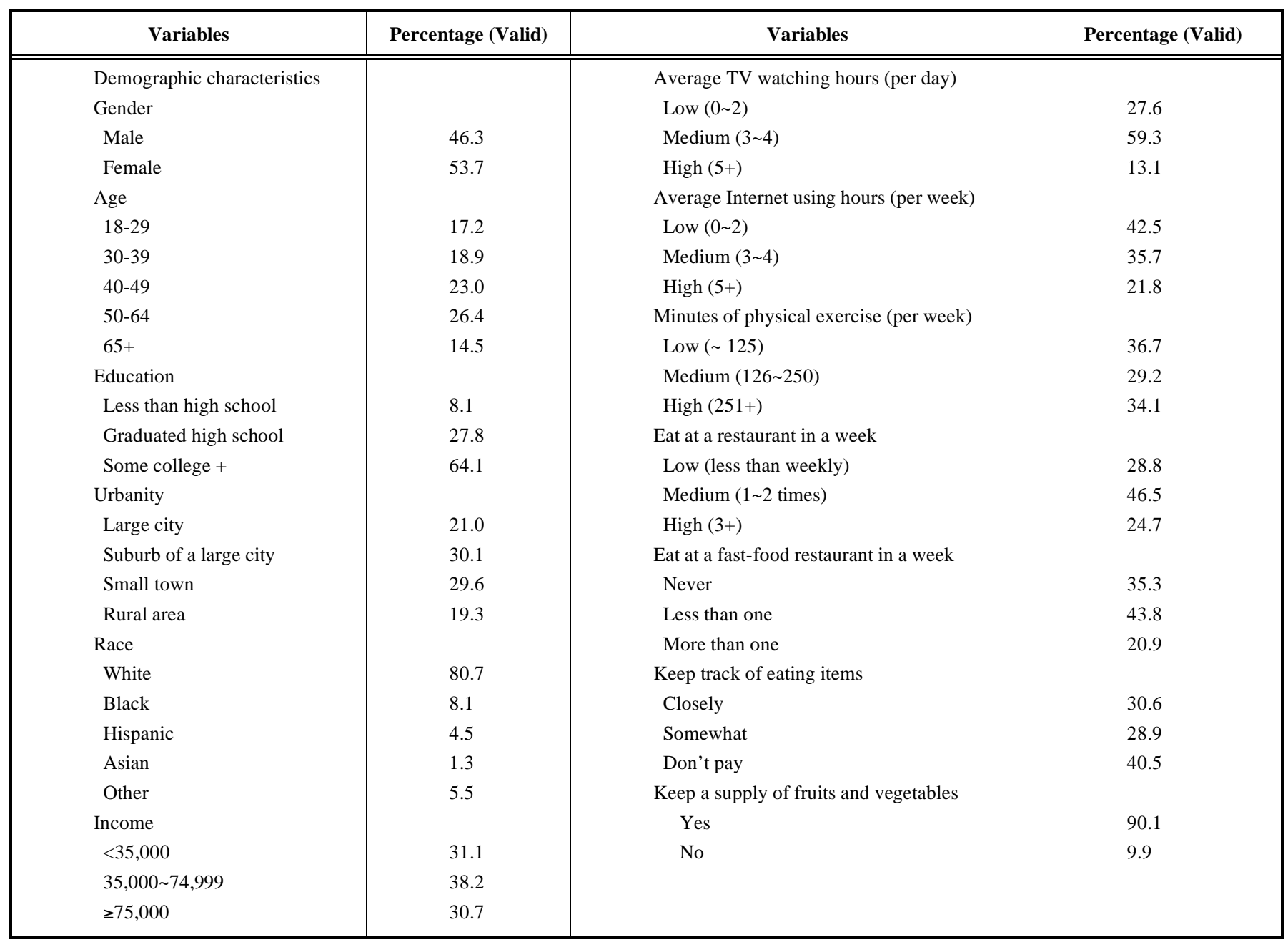

The main outcome variables are overweight or obese $(25 \leq \mathrm{BMI} \leq 30)$ and obese $(\mathrm{BMI} \geq 30)$ levels that were developed by BMI. Body Mass Index (BMI, $\mathrm{kg} / \mathrm{m}^{2}$ ) was calculated from self-reported height and weight. In addition, underlying predictors of being overweight and obesity were blocked into three factors: (1) demographic \& socioeconomic status factors, (2) sedentary lifestyle factors, (3) dietary behavior factors.

\section{Socioeconomic Status \& Sociodemographic Factor Mea- sures}

The socioeconomic status factors being studied are education and income, based on the self-reported information from the poll. Education is measured as respondents' final education and is grouped into three-categories: less than high school; graduated high school; some college. Income is measured as the total combined income, before tax, for all the members of household in the preceding year, and also is grouped into three categories: $\$ 1$ through $\$ 34,999 ; \$ 35,000$ through $\$ 74,999 ; \$ 75,000$ or more. More refined categories of education and income showed similar result trends. Age is grouped into five categories: 18 through 29 years; 30 through 39 years; 40 through 49 years; 50 through 64 years; 65 years or older. Other demographic variables being studied include sex (male vs. female), race (White, Black, Hispanic and Asian), and urbanity of residence (large city, suburb of a large city, small town, and rural area).

\section{Sedentary Lifestyle Factor Measures}

Television watching hours is measured into respondents' average television watching hours per day and is grouped into three categories: low (0 2 hours), medium (3 4 hours), and (5 or more hours). Average Internet use hours per week are also categorized into three groups: low (0 2), medium (3 4), and high (5 or more hours). Amount of physical exercise is coded using three categories based on the minutes of exercise done per a week: low (less than 125 minutes), medium (126 250 minutes), and high (more than 251 minutes).

\section{Dietary Behavior Factor Measures}

Dietary behavior factors were composed on the answers to questions regarding how often the respondent ate at a fast food restaurant and full-service restaurant in a week, whether the respondent kept track of his or her eaten items and if they continued to eat fruits and vegetables on a regular basis. Full service restaurant visits were grouped into three categories: low (less than weekly), medium (1 2 times), and high (more than 3 times). Visiting frequencies of fast food restaurants were divided into three categories such as never, less than one, and more than one times per a week. 
Table 2. Prevalence of Obesity by Socioeconomic Status Factors

\begin{tabular}{|c|c|c|c|c|c|c|}
\hline \multirow[b]{2}{*}{ Factors } & \multicolumn{3}{|c|}{ Education (Valid Percentage) } & \multicolumn{3}{|c|}{ Income (Valid Percentage) } \\
\hline & Less than high & Grad high & Some college & $<35,000$ & $35,000 \sim 75,000$ & $>75,000$ \\
\hline \multirow{3}{*}{ Obese } & 18.5 & 22.9 & 14.1 & 20.4 & 19.6 & 10.0 \\
\hline & 100 & 100 & 100 & 100 & 100 & 100 \\
\hline & \multicolumn{3}{|c|}{$\chi^{2}=12.452(p<.01)$} & \multicolumn{3}{|c|}{$\chi^{2}=15.245(p<.01)$} \\
\hline
\end{tabular}

\section{Data Analysis}

Final data are weighted using demographic information from the Census Bureau's most recent Current Census Population Survey to adjust for sampling and non-sampling deviations from population values based on age, race, sex, and education. All of the analyses reported below are based on the post-stratification adjusted data (Silver, Mulvey, \& Swanson, 2002). Logistic regression was employed to model the effects of socioeconomic status and socio-demographic factors, sedentary lifestyle and dietary characteristics on being overweight and obesity.

The effects of each independent variable being studied on overweight and obesity rate were analyzed separately. A series of multiple predictor models were then estimated. First, the relative probability of becoming overweight and obese was estimated for income and education groups (socioeconomic variables) and socio-demographic variables (gender, age, urbanity and race) together. Second, the behavioral risk factors (sedentary lifestyle and dietary behaviors) were added hierarchically to the baseline model to investigate how additional factors affect the prevalence of overweight and obesity in combination with the prior socioeconomic status and socio-demographic variables.

\section{RESULTS}

Table 1 presents information on the demographic \& socioeconomic conditions and patterns of risk behaviors among the national representative adult sample. A significant portion of sample respondents were well socioeconomically distributed. Income levels are evenly distributed. About 64 percent of the sample had experience of college education, whereas eight percent of respondents reported educational background of "less than high school". Socio-demographic variables of gender, age, urbanity are also evenly distributed, but the White population was oversampled when compared to the other racial groups.

The majority of respondents watched television three to four hours a day (59.3\%), while mean hours of Internet use per week is still less than 2 hours. The average visits of full service restaurants and fast food restaurants were 1 2 times and less than one time per a week respectively. About twothirds of the respondents exercise more than two hours per a week.

The distribution of the dichotomous variable of being obese $(\mathrm{BMI} \geq 30)$ or not $(\mathrm{BMI}<30)$ was determined significantly by educational attainment and annual household income. Table 2 shows an inverse relationship between education attainment and obesity level. A similar pattern was also found between household income and being obese. In sum- mary, persons with the least amount of education as well as the lowest income were significantly more likely to be obese.

The results of model 1 at Table 3 provide evidences of statistical associations between a range of socioeconomic conditions and the probability of becoming overweight $(25 \leq \mathrm{BMI}<30)$ and obese $(\mathrm{BMI} \geq 30)$. Statistical associations were found between the risk of becoming overweight/obese and various socio-demographic (e.g., gender, age, urbanity, and race) and socioeconomic conditions (e.g., income and education). As we get older, we are more likely to be overweight. For example, persons in their fifties are almost four times more likely to be overweight than teenagers (odd ratio $=3,990$, reference group: age of 18 29). Residents in rural areas are two times more likely to be overweight when compared to city dwellers (odd ratio=1.959). No relationships were found between education attainment and being overweight, whereas individuals of higher annual incomes $(>\$ 75,000)$ are half as likely to become overweight compared to those with having lower annual incomes $(<35,000)$. Probabilities of becoming overweight were higher among black (odd ratio $=2.119$ ) and Hispanic (odd ratio $=2.179$ ) than White population. Model 1 of Table 3 also provides statistically significant associations between the risk of becoming obese and unique conditions a person belongs to. Probabilities of becoming obese are generally attenuated when compared with becoming overweight across all significant variables. Gender, educational attainment, and urbanity of the respondents are not significantly associated with being obese in Model 1. The ineffectiveness of education attainment on overweight or obesity suggests that a portion of the association between household income level and overweight and obesity in Model 1 operates through the respondents' educational attainment. Regardless of model specifications, however, results showed that respondents who are older, having lower income and non-White had higher risks of becoming overweight or obese. These results answer the RQ 1.

When sedentary life style variables were incorporated into the model (Model 2), most part of the influences of socioeconomic conditions remained relatively unchanged, whereas effects of race and income disappeared when obesity was a dependent variable. In consistent with prior findings of relationship between television viewing and obesity prevalence (Campbell, Crawford, \& Jackson, 2002), a person with heavy TV watching habit has higher risks of becoming overweight (odd ratio $=2.482$ ) and obese (odd ratio=3.100). Compared with television watching hours, average Internet usage did not exert any significant effect on being overweight or obesity. Different involvement levels and using patterns employed for Internet usage give us a clue to the 
Table 3. Effects of Individual Risk Behavioral Factors on Overweight and Obesity: National survey of ABC News/Time Magazine Obesity Poll, May 2004 ( $N=1,202)$

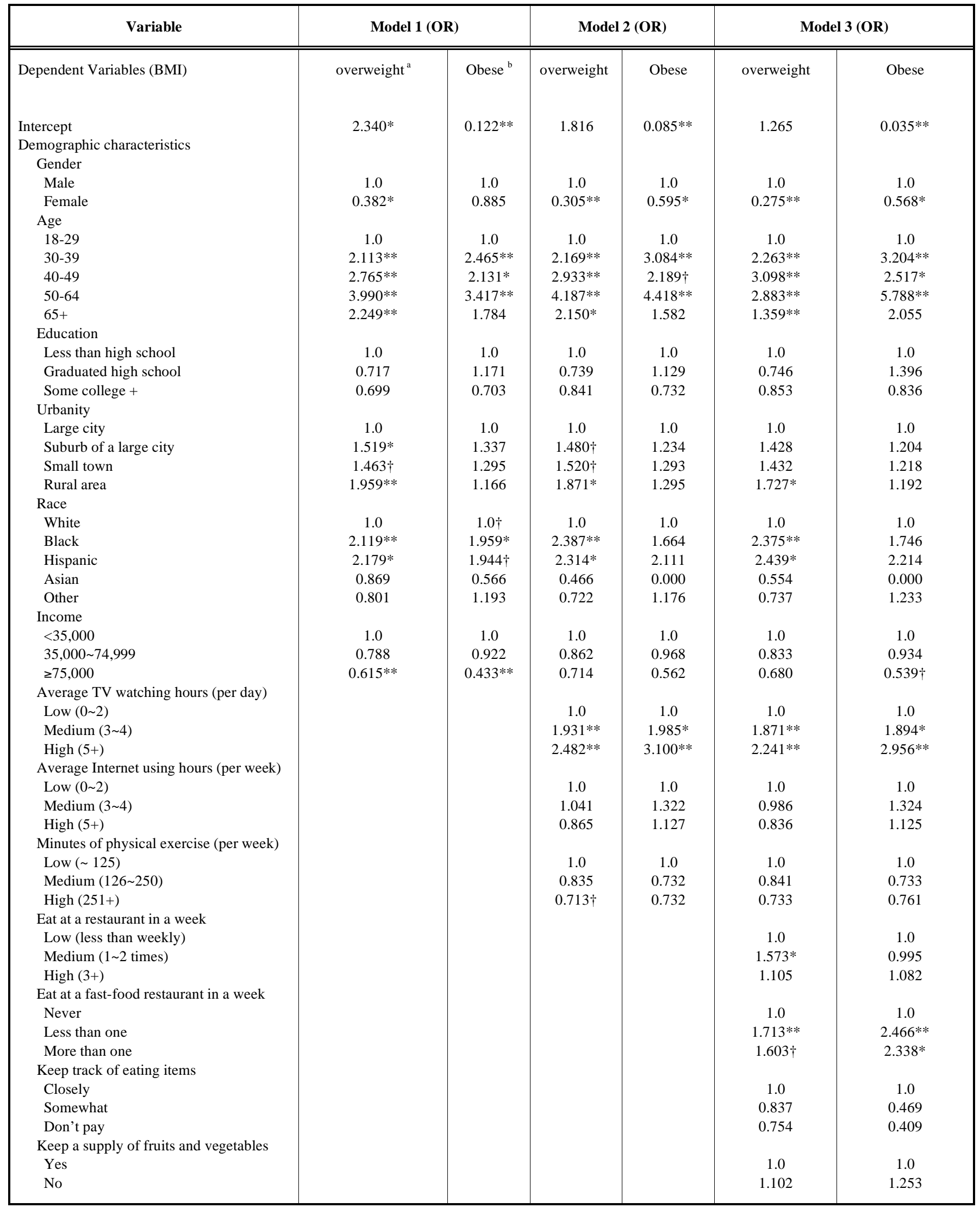


never-users, compared with 1.6 times of probability of becoming overweight. These results answer the RQ 2.

\section{DISCUSSIONS}

The current study contributes to the literature because it uses the theory-driven approach to verify the influence of a few critical variables on the prevalence of overweight/obesity, and suggests solid empirical evidences that guide constructive solutions to curve the vicious cycle of unhealthy lifestyle and gaining weight. Other researches have evaluated the fragmented effects of biological, social and behavioral factors on gaining weight without comprehensive considerations of critical factors engendering a national problem of obesity.

People with low SES and sedentary lifestyle showed greater chances to be overweight or obese when they chronically follow unhealthy diet. In addition, at the model 3 of the table 3, when sedentary lifestyle and dietary pattern were incorporated into the baseline model of only considering socioeconomic status, the effects of SES (e.g., household income) and socio-demographic factors were attenuated. Whereas individual behavioral factors such as average TV watching hours and frequencies of visiting fast food restaurants became to have significant logit coefficients on becoming overweight and obese. We may assume that socioeconomic and socio-demographic variables are underlying latent variables that have indirect effects on developing overweight and obesity. Socioeconomic factors are likely to work through other proximal factors to create weight differentials. More sophisticated statistical analyses are recommended to verify indirected effects of critical variables leading to be overweight or obese.

In consistent with WHO consultations on obesity (1998), the fundamental causes of becoming obese are found to be sedentary lifestyle and energy-dense diet. The results at table 3 show that sedentary lifestyle of television watching and frequent visits of fast-food restaurant exert significant effects on overweight and obesity development. The insignificant association between physical exercise and gaining weight may be better answered when we add the consistency of exercise program over some period of time in combination with the analysis of the decrease in work-related physical activity.

Extended TV watching hours have double effects on gaining weight; pure inactivity of a couch potato lifestyle on the one hand as well as the influence of television advertising on health attitudes and behaviors on the other hand. Comprehensive and effectively designed public health campaign interventions are anticipated to counter fast food companies' organized and well-funded marketing campaigns. The high odd ratios of high-level television watchers to be overweight $(\mathrm{OR}=2.24, p<0.01)$ and obese $(\mathrm{OR}=2.96$, $p<0.01)$ support the negative influences of TV commercials on maintaining healthy weight. If we increase social marketing activities promoting healthy lifestyles (e.g., eat more fruits and vegetables) countering fast-food consumptions and sedentary life styles, the rapid increase of obesity rate could be decreased. Social marketing may offer principles and techniques for overcoming the challenges the obesity epidemic highlights. Basically, governmental entities and NGOs should be involved in developing obesity related policies and reinforcing regulations especially on TV commercials launched by fast food industries.

\section{REFERENCES}

Adler, N. F., Boyee, T., \& Chesney, M. A. (1994). Socioeconomic status and health: the challenge of the gradient. American Psychology, 49, 15-24.

AOA. (2004). AOA fact sheet: American Obesity Association. Retrieved July 1, 2008 from the World Wide Web: http://www.obesity.org/information/factsheets.asp.

Barns, M. A., \& Schoenborn, C. A. (2003). Physical activity among adults: United States. National Center for Health Statistics. Advanced Data, 333.

Calle, E. E., Thun, M. J., Petrelli, J. M., Rodriguez, C., \& Heath, C. W. J. (1999). Body mass index and mortality in a prospective cohort of U.S. adults. New England Journal of Medicine, 341(15), 10971105 .

Campbell, K., Crawford, D., \& Jackson, M. (2002). Family food environments of 5 and 6-year-old children: Does socioeconomic status make a difference? Asia Pacific Journal of Clinical Nutrition, 11, 553-561.

Caspersen, C. J., Pereira, M. A., \& Curran, K. M. (2000). Changes in physical activity patterns in the United States by sex and cross-sectional age. Medical Science Sports Exercise, 32, 1601-1609.

Chambliss, H. O., Finley, C. E., \& Blair, S. N. (2004). Attitudes toward obese individuals among exercise science students. Medicine \& Science in Sports \& Exercise, 36, 468-474.

Dietz, W. H., \& Gortmaker, S. L. (1985). Do we fatten our children at the television set? Obesity and television viewing in children and adolescents. Paediatrics, 75(5), 807-812.

Flegal, K. M., Carroll, M. D., Ogden, C. L., \& Johnson, C. L. (2002). Prevalence and trends in obesity among US adults, 1999-2000. Journal of American Medical Association, 288, 1723-1727.

Flegal, K. M., Graubard, B. I., Williams, D. F., \& Gail, M. H. G. (2005). Excess deaths associated with underweight, overweight, and obesity. Journal of American Medical Association, 293(15), 18611867.

French, S. A., Harnack, L., \& Jeffrey, R. W. (2000). Fast food restaurant use among women in the pound of prevention study: dietary, behavioral, and demographic correlates. International Journal of Obesity, 24, 1353-1359.

French, S. A., Story, M., \& Jeffrey, R. W. (2001). Environmental influences on eating and physical activity. Annual Review of Public Health, 22, 309-335.

Goodman, E., Slap, G. B., \& Huang, B. (2003). The public health impact of socioeconomic status on adolescnet depression and obesity. American Journal of Public Health, 93(11), 1844-1850.

Harver-Berino, J., Pintauro, S., \& Gold, E. (2002). The feasibility of using Internet support for the maintenance of weight loss. Behavior Modification, 26(1), 101-116.

Levy, A. S., Fein, S. B., \& Stephenson, M. (1993). Nutrition knowledge levels about dietary fats and cholesterol. Journal of Nutrition Education, 25, 60-66.

Link, B. G., \& Phelan, J. (1995). Social conditions as fundamental causes of disease. Journal of Health and Behavior(Extra Issue), 80-94.

Manson, J. E., Stamfer, M. J., Hennekens, C. H., \& Willett, W. C. (1987). Body weight and longevity: A reassessment. Journal of American Medical Association, 257, 353-358.

McGinnis, M. J., \& Foege, W. H. (1993). Actual causes of death in the United States. Journal of American Medical Association, 270, 2207-2212.

MMWR. (2004). Youth Risk Behavioral Surveillance - United States. De partment of Health and Human Services, 53(No SS-2).

Mokdad, A. H., Ford, E. S., Bowman, B. A., Diez, W. H., Vinicor, F. Bales, V. S., \& Marks, J. S. (2003). Prevalence of obesity, diabetes, and obesity-related health risk factors. Journal of American Medical Association, 289(1), 76-79.

Pastor, P. N., Makuc, D. M., Reuben, C., \& Xiz, H. (2002). Chartbook on trends in the health of Americans. In Health, United States. Hyattsville, MD: National Center for Health Statitics.

Pi-Sunyer, \& Xavier, F. (1999). Comobidities of overweight and obesity: current evidence and research issures. Medicine \& Science in Sports \& Exercise, 31(11), S602-S608.

Racette, S. B., Deusinger, S. S., Strube, M. J., Highstein, G. R., \& Deusinger, P. T. (2005). Weight changes, exercises, and dietary patterns 
during freshemen and sophmore years of college. Journal of American College Health, 53(6), 245-251.

Saba, A., Vassallo, M., \& Turrini, A. (2000). The role of attitides, intentions and habits in the actual consumption of fat containing foods in Italy. European Journal of Clinical Nutrition, 54, 540-545.

Silver, E., Mulvey, E. P., \& Swanson, J. W. (2002). Neighborhood structural characteristics and mental disorder: Faris and Dunham revisited. Social Science \& Medicines, 55, 1457-1470.

Sjostrom, C. J., Lissner, L., \& Sjostrom, L. (1997). Relationships between changes in body composition and changes in cardiovascular risk factors: The SOS intenvention study. Obesity Research, 5(6), 519530.

Strum, R., \& Wells, K. (2001). Does obesity contribute as much to mordidity as poverty or smoking? Public Health, 115, 229-295.
Van Cauter, E., \& Spiegel, K. (1999). Sleep as a mediator of the relationship between socioeconomic status and health: a hypothesis. Annals of the New York Academy of Sciences, 896, 254-261.

WHO. (1998). Obesity: preventing and managing the global epidemic. Geneva: World Health Organization.

Willett, W. C., Manson, J. E., Stampfer, M. J., Golditz, G. A., Rosner, B., Speizer, F. E., Et Al. (1995). Weight, weight change and coronary heart disease in women: Risk within the normal weight range. Journal of American Medical Association, 273, 461-465.

Winkleby, M. A., Fortmann, S. P., \& Barrett, D. C. (1990). Social class disparities in risk factors for disease: eight year prevalence patterns by level of education. Preventive Medicine, 19, 1-12.

Yach, D. (2004). Complex and controversial causes for the obesity epidemic. International Journal of Medical Marketing, 4(3), 288-290.

Received: April 08, 2008

Revised: May 31, 2008

Accepted: June 09, 2008

(c) Taejin Jung; Licensee Bentham Open.

This is an open access article distributed under the terms of the Creative Commons Attribution License (http://creativecommons.org/licenses/by/2.5/), which permits unrestrictive use, distribution, and reproduction in any medium, provided the original work is properly cited. 